PSA DENSITY, a lower percentage of positive biopsies, and with earlier-stage disease than patients not taking statins. Although not all differences were statistically significant, almost all clinical presentation parameter comparisons favored statin users; for example, the 8-year bPFS was $97.0 \%$ for statin users compared to $94.3 \%$ for those not taking statins $(P=0.398)$. In addition, atorvastatin seemed to have a greater effect on bPFS than other statins.

The authors suggest that the lipophilicity of atorvastatin, allowing easier penetration of cell membranes, might explain its enhanced effect compared to that of other statins. They conclude that a prospective trial of statins combined with conventional prostate cancer therapy is warranted, especially as cardiovascular disease is a major cause of death in patients with this condition.

Jim Casey

Original article Moyad MA et al. (2005) Statins, especially atorvastatin, may favorably influence clinical presentation and biochemical progression-free survival after brachytherapy for clinically localized prostate cancer. Urology 66: 1150-1154

\section{Preoperative behavioral training reduces incontinence after prostatectomy}

Incontinence is a common consequence of radical prostatectomy, but postoperative behavioral training has been shown to improve continence after prostatectomy. Researchers in Alabama have conducted a randomized trial to evaluate the effect of preoperative biofeedbackassisted behavioral training on incontinence in the 6 months following surgery, on 125 men who underwent elective radical prostatectomy between 1996 and 2001.

Men assigned to the intervention group received a single training session, in which they learned pelvic-floor muscle control and were instructed in daily pelvic-floor muscle exercise. Men in the control group were given brief verbal instructions to interrupt their urine stream while voiding, plus whatever instructions were given by the surgeon as part of usual care. Median time to continence in the intervention group was 3.5 months, whereas $<50 \%$ of the control group had achieved continence by 6 months after surgery. After 6 months, the proportion of patients suffering severe or continual leakage was $5.9 \%$ in the intervention group and $19.6 \%$ in the control group. Incidence of stress incontinence was also markedly lower in the intervention group.

The authors conclude that preoperative training can reduce the duration and severity of incontinence after prostatectomy, and that further research is required to determine the optimal time to begin training and to evaluate the usefulness of biofeedback. They recommend that urologists consider either referring patients to a continence center for pelvic-floor muscle training, or incorporate such training into their office practice.

\section{Jim Casey}

Original article Burgio KL et al. (2006) Preoperative biofeedback assisted behavioral training to decrease postprostatectomy incontinence: a randomized, controlled trial. JUrol 175: 196-201

\section{GLOSSARY}

PSA DENSITY

The relationship of serum PSA level to the size of the prostate; the PSA value is divided by the prostate volume 